\section{Análisis focalizado de calidad de vida en adultos}

\section{Focused assessment of quality of life in adults}

\section{Sr. Editor:}

En un reciente estudio sobre la calidad de vida en adultos ${ }^{1}$, los autores hacen una importante contribución descriptiva sobre las características de la misma. Pero pensamos que el diseño de su estudio redujo el poder estadístico y la magnitud de sus resultados, pues las diferencias entre los grupos de edad deben plantearse mejor como hipótesis direccionales. Esto es debido a la aparente tendencia de medias que reportaron en las Tablas 6 y 7. Por lo tanto, la naturaleza difusa del ANOVA "ómnibus" (o no focalizado) aplicado por los autores no permitió revelar otros resultados que complementan la importancia de su estudio ${ }^{2-4}$. Aunque el ANOVA aplicado claramente identifica que la edad es una fuente de variación más allá del error de muestreo, se requiere más precisión en su análisis, y esto es un problema al aplicar pruebas estadísticas generales o de tipo "ómnibus" 2,4 .

Para examinar la direccionalidad de las diferencias, se examinaron las medias reportadas en sus Tablas 6 y 7, mediante un análisis focalizado $o^{4}$, planteando que las diferencias de edad tendrían una tendencia decreciente. Este planteamiento se respalda por la observación de las medias reportadas en sus Tablas, pero que no fueron formalmente contrastadas. La presentación cuantitativa de este procedimiento se hace con un contraste ortogonal, que permite separar la variabilidad entre grupos total, de la variabilidad proveniente del contraste. En este re-análisis, se probarán dos tendencias: lineal $(+1,0,-1)$ y cuadrática $(-1,2$, -1 , que describen dos formas diferentes de tendencia de las medias. Los resultados se presentan en nuestra Tabla 1, con el estadístico de contraste para la tendencia cuadrática y lineal, e indicadores de magnitud del efecto ${ }^{4,5}$ :

Hallamos lo siguiente: Primero, nuestros resultados muestran que, comparado con el ANOVA general de los autores, el planteamiento de contrastes produjo valores $\mathrm{F}$ más elevados, indicando que la evidencia contra la hipótesis nula es más fuerte. Segundo, la variación entre los grupos se explica por diferentes tipos de tendencias (en este análisis, lineal o cuadrático). Esto puede comprobarse porque la magnitud del efecto de un contraste $\left(r_{\text {efec }}\right)$ no es similar en los dominios de $\mathrm{CV}$; por ejemplo, en el dominio social predomina la tendencia lineal mientras que en el dominio psicológico predomina la tendencia cuadrática. Tercero, la ponderación por importancia enfatiza más las diferencias que se observan en los dominios sin ponderar.

Tabla 1. ANOVA (para edad y CV) con contrastes cuadrático y lineal

\begin{tabular}{|c|c|c|c|c|c|}
\hline & \multirow{2}{*}{$\begin{array}{c}\text { F original } \\
\text { (Urzúa et al., 2013) }\end{array}$} & \multicolumn{2}{|c|}{ Tendencia cuadrática } & \multicolumn{2}{|c|}{ Tendencia lineal } \\
\hline & & $\boldsymbol{F}$ & $\boldsymbol{r}_{\text {efec }}$ & $\boldsymbol{F}$ & $\boldsymbol{r}_{\text {efec }}$ \\
\hline \multicolumn{6}{|l|}{ Sin ponderación } \\
\hline $\begin{array}{l}\text { Dominio físico } \\
\text { (gl: } 1,1224)\end{array}$ & $9,222^{* *}$ & $11,73^{* *}$ & 0,097 & $17,78^{* * *}$ & 0,11 \\
\hline $\begin{array}{l}\text { Dominio psicológico } \\
\text { (gl: } 1,1225)\end{array}$ & $5,537^{*}$ & $9,57 * *$ & 0,080 & 1,28 & 0,032 \\
\hline $\begin{array}{l}\text { Dominio social } \\
\text { (gl: } 1,1226)\end{array}$ & $13,554^{* *}$ & $5,795^{*}$ & 0,068 & $20,70^{* * *}$ & 0,129 \\
\hline \multicolumn{6}{|c|}{ Ponderados por importancia } \\
\hline $\begin{array}{l}\text { Dominio físico } \\
\text { (gl: } 1,1224)\end{array}$ & $8,173 * *$ & $13,204^{* * *}$ & 0,103 & 2,809 & 0,048 \\
\hline $\begin{array}{l}\text { Dominio psicológico } \\
\text { (gl: } 1,1225)\end{array}$ & $6,578^{*}$ & $11,93^{* * *}$ & 0,098 & 0,92 & 0,027 \\
\hline $\begin{array}{l}\text { Dominio social } \\
\text { (gl: } 1,1225)\end{array}$ & $15,087^{* * *}$ & $8,25^{* *}$ & 0,081 & $21,13^{* * *}$ & 0,130 \\
\hline
\end{tabular}

CV: Calidad de Vida. F: prueba F, ANOVA. $r_{\text {efec }}$ : magnitud del efecto. 
Estos resultados, formalmente identificados, abren otra vía de interpretación pues las tendencias detectadas indican patrones de cambio reconocibles (cuadrático o lineal). La teoría, que bien conocen los autores, es la pieza fundamental para aportar razonables explicaciones a los contrastes hallados y ayudará a discernir finalmente la naturaleza de la variación y configurar otras preguntas de investigación. Sin embargo, debemos hacer notar que el re-análisis efectuado fue de carácter a posteriori, cuando una mejor metodología debió ser su planteamiento a priori $i^{3,6}$.

César Merino-Soto ${ }^{1}$, Marisol Angulo-Ramos ${ }^{2}$ ${ }^{1}$ Instituto de Investigación de Psicología, Universidad de San Martín de Porres. Lima, Perú. ${ }^{2}$ Práctica Privada. Lima, Perú.

\section{Referencias}

1. Urzúa A, Cortés K, Maita C, Osorio K, Caqueo-Urizar A. La valoración de la importancia en el autoreporte de la calidad de vida en la adultez. Rev Med Chile 2013; 141: 1010-8.

2. Rosnow RL, Rosenthal R: Computing contrasts, effect sizes, and counternulls on other people's published data: General procedures for research consumers. Psychol Methods 1996; 1: 331-40.

3. Rosnow RL, Rosenthal R: Focused tests of significance and effect size estimation in counseling psychology. J Couns Psychol 1988; 35: 203-8.

4. Rosnow RL, Rosenthal R, Rubin DB. Contrasts and correlations in effect-size estimation. Psychological Sci. 2000; 11 (6): 446-53.

5. Rosnow RL, Rosenthal R. Effect sizes for experimenting psychologist. Can J Exp Psychol 2003; 57 (3): 221-37.

6. Rosnow RL, Rosenthal R.Contrast and interactions redux: Five easy pieces. Psychol Sci 1996; 7: 253-7.

Nota del Editor: Los autores aludidos en esta carta declinaron su derecho a réplica.

Correspondencia a:

César Merino-Soto. Calle Filiberto Romero 430, Chorrillos - Lima 09, Perú.

sikayax@yahoo.com.ar 